\title{
FTY720 inhibited proinflammatory cytokine release and osteoclastogenesis induced by Aggregatibacter actinomycetemcomitans
}

\author{
Hong Yu ${ }^{1 *}$, Bethany A. Herbert ${ }^{1}$, Michael Valerio ${ }^{1}$, Leigh Yarborough ${ }^{2}$, Li-Chien Hsu ${ }^{2}$ and Kelley M. Argraves ${ }^{3}$
}

\begin{abstract}
Background: Periodontitis is a bacteria-driven inflammatory bone loss disease. Previous studies showed that the oral pathogen Aggregatibacter actinomycetemcomitans (A. actinomycetemcomitans) stimulated the generation of sphingosine 1 phosphate (S1P). In addition, S1P signaling regulated the migration of osteoclast precursors and affected osteoclastogenesis. Furthermore, treatment with FTY720 (also called fingolimod, a modulator of multiple S1P receptors) alleviated osteoporosis and suppressed arthritis in animals. This study determined the effect of FTY720 on proinflammatory cytokine production and osteoclastogenesis in murine bone marrow cells with or without $A$. actinomycetemcomitans stimulation.
\end{abstract}

Methods: Murine bone marrow-derived monocytes and macrophages (BMMs) were treated with vehicle ethanol or FTY720, and were either unstimulated or stimulated for 0.5 to $6 \mathrm{~h}$ with A. actinomycetemcomitans. The protein levels of interleukin (IL)-1 $\beta, I L-6$, and tumor necrosis factor (TNF)- $a$ in the media of BMMs were quantified by enzyme-linked immunosorbent assay (ELISA). Protein expressions, including phosphorylated phosphoinositide 3-kinase (p-PI3K), p-Akt, p-extracellular signal-regulated kinase (p-ERK), PI3K, Akt, and ERK were evaluated by Western blot. In addition, murine bone marrow-derived pre-osteoclasts were treated with macrophage colony-stimulating factor (M-CSF) and receptor activator of nuclear factor kappa-B ligand (RANKL) for three days. Then the cells were treated with either vehicle or FTY720 and were either unstimulated or stimulated with A. actinomycetemcomitans for 4 to $24 \mathrm{~h}$. Control cells were treated with M-CSF alone with or without bacterial stimulation. Osteoclasts were stained by tartrate-resistant acid phosphatase (TRAP) staining. The mRNA levels of osteoclastogenic factors, including nuclear factor of activated T-cells cytoplasmic calcineurin-dependent 1 (Nfatc1), cathepsin K (Ctsk), acid phosphatase 5 (Acp5), osteoclast-associated receptor (Oscar), and RANKL were quantified by quantitative real-time polymerase chain reaction (PCR).

Results: FTY720 dose-dependently inhibited IL-1 $\beta$, IL-6, and TNF-a protein levels induced by A. actinomycetemcomitans in BMMs compared with controls. Additionally, FTY720 attenuated p-PI3K, p-Akt, and p-ERK expressions induced by A. actinomycetemcomitans. Furthermore, FTY720 suppressed osteoclastogenesis in bone marrow-derived pre-osteoclasts with or without bacterial stimulation and reduced the mRNA levels of Nfatc1, Ctsk, Acp5, and Oscar, but not RANKL in bone marrow-derived pre-osteoclasts.

Conclusion: FTY720 inhibited proinflammatory cytokine production and suppressed osteoclastogenesis, supporting FTY720 as a potential therapy for inflammatory bone loss diseases.

Keywords: FTY720, Sphingosine-1-phosphate, Periodontitis, Cytokine, Osteoclastogenesis, Aggregatibacter actinomycetemcomitans

\footnotetext{
* Correspondence: yuho@musc.edu

'Department of Oral Health Sciences and the Center for Oral Health Research, Medical University of South Carolina, 173 Ashley Avenue, Charleston, SC 29425, USA

Full list of author information is available at the end of the article
}

\section{Ciomed Central}

(c) 2015 Yu et al. This is an Open Access article distributed under the terms of the Creative Commons Attribution License (http://creativecommons.org/licenses/by/4.0), which permits unrestricted use, distribution, and reproduction in any medium, provided the original work is properly credited. The Creative Commons Public Domain Dedication waiver (http:// creativecommons.org/publicdomain/zero/1.0/) applies to the data made available in this article, unless otherwise stated. 


\section{Background}

Periodontitis is a bacteria-driven inflammatory bone loss disease. A. actinomycetemcomitans is an oral pathogen associated with localized aggressive periodontitis. Oral bacterial pathogens initiate a host inflammatory response, leading to proinflammatory cytokine production, progressive alveolar bone loss, and subsequent tooth loss [1]. One of the hallmarks of periodontitis is inflammation-induced osteoclastogenesis. While observed, the mechanisms associated with the inflammatory bone loss response induced by oral pathogens have not been completely elucidated.

Previously, we showed that $A$. actinomycetemcomitans stimulated the generation of sphingosine-1-phosphate (S1P) in RAW 264.7 cells, a murine monocyte and macrophage cell line [2]. S1P is a bioactive sphingolipid, which can be generated in most mammalian cells by various stimuli [3]. Intracellular S1P can be exported to extracellular space by specific transporters. S1P binds to five G protein-coupled S1P receptors (S1PR1-5) on the plasma membrane initiating various cellular signaling pathways $[4,5]$. S1P signaling plays an important role in regulating cell growth, proliferation, adhesion, chemotaxis, cytokine production, and bone homeostasis $[4,6-8]$.

S1P signaling affects the pathogenesis of many diseases, including inflammatory diseases, arthritis, and osteoporosis [9-11]. The synovial fluid of rheumatoid arthritis patients exhibits significantly higher levels of S1P than their noninflammatory osteoarthritis counterparts [12]. Additionally, S1P signaling controls the migration of monocytes and macrophages (osteoclast precursors) from blood circulation to bone tissues $[7,8]$ and stimulates the generation of RANKL, an osteoclastogenic factor, which affects bone homeostasis [13]. Furthermore, high circulating S1P levels observed in postmenopausal women are positively correlated with their bone resorption markers [14]. However, the roles of S1P signaling in modulating proinflammatory cytokine release and osteoclastogenesis induced by oral pathogens has not been defined.

FTY720 \{2-amino-2-[2-(4-octylphenyl) ethyl]-1,3-propanediol hydrochloride\} was synthesized by structural modification of myriocin, a fungal metabolite from Isaclaria sinclarii, a traditional herb used in Eastern medicine [15]. FTY720 is phosphorylated to p-FTY720 by sphingosine kinase. P-FTY720 functions as a noncompetitive inhibitor of multiple S1PRs [16, 17]. P-FTY720 blocks S1P signaling by inducing the internalization and partial degradation of S1PRs [16]. FTY720 has been shown as a potent immune suppressant with low toxicity. It has been used in clinical trials to treat relapsing multiple sclerosis and prevent the rejection of renal transplant $[18,19]$. Additionally, treatment with FTY720 alleviated ovariectomy-induced osteoporosis in mice [7] and attenuated arthritis in mice induced by arthrogenic anti-collagen II antibodies cocktail and lipopolysaccharide, as compared with control treatment [20].

Monocytes and macrophages are major sources of proinflammatory cytokines in chronic inflammatory diseases. During inflammatory pathogenesis, bacterial pathogens activate various cellular signaling cascades including phosphoinositide 3-kinase (PI3K)-Akt (also known as protein kinase $B$ ), mitogen-activated protein kinases (MAPKs), and nuclear factor kappa-B (NF-kB) pathways. The MAPKs include the extracellular signalregulated kinase (ERK), c-Jun N-terminal kinase (JNK), and p38 MAPK. Activation of these signaling pathways results in proinflammatory cytokine release. Additionally, monocytes and macrophages are osteoclast precursors, which can fuse to form multinucleated mature osteoclasts leading to bone loss [21]. Osteoclastogenesis is regulated by cytokines, including M-CSF and RANKL, which are essential for osteoclastogenesis [22]. M-CSF is essential for the survival and proliferation of osteoclast progenitors and macrophages, while RANKL is important for the differentiation of osteoclasts [22]. Additionally, nuclear factor of activated T-cells cytoplasmic calcineurin-dependent 1 (Nfatc1) is considered the master transcription factor during osteoclast differentiation [23]. Nfatc1 regulates transcription of many osteoclastogenic genes, including cathepsin K (Ctsk), acid phosphatase 5 (Acp5), and osteoclast-associated receptor (Oscar) [24, 25]. Although previous studies demonstrated that FTY720 inhibited immune response and attenuated bone loss [7, 18-20], the mechanisms associated with the roles of FTY720 on modulating inflammatory diseases and bone loss diseases have not yet been completely clarified. In this study, we elucidated the mechanisms associated with the effects of FTY720 in proinflammatory cytokine production and osteoclastogenesis with or without $A$. actinomycetemcomitans stimulation.

\section{Results \\ FTY720 dose-dependently inhibited IL-1 $\beta$, IL-6, and TNF- $\alpha$ protein levels induced by $A$. actinomycetemcomitans in BMMs}

Because FTY720 inhibited inflammatory response in previous in vivo studies [26], we hypothesized that FTY720 regulated the proinflammatory responses induced by $A$. actinomycetemcomitans. To test our hypothesis, BMMs derived from C57BL/6 mice were treated with either vehicle (ethanol) or FTY720 (2 to $8 \mu \mathrm{M})$ for $30 \mathrm{~min}$. Then the cells were either unstimulated or stimulated with $A$. actinomycetemcomitans $(1.5 \mathrm{CFU} /$ cell $)$ for $6 \mathrm{~h}$. The protein levels of IL-1 $\beta$, IL6 , and TNF- $\alpha$ in cell culture media were quantified. As shown in Fig. 1a-c, FTY720 significantly suppressed IL-1 $\beta$, IL- 6 , and TNF- $\alpha$ expressions induced by $A$. actinomycetemcomitans in a dose-dependent manner compared with the control treatment. FTY720 

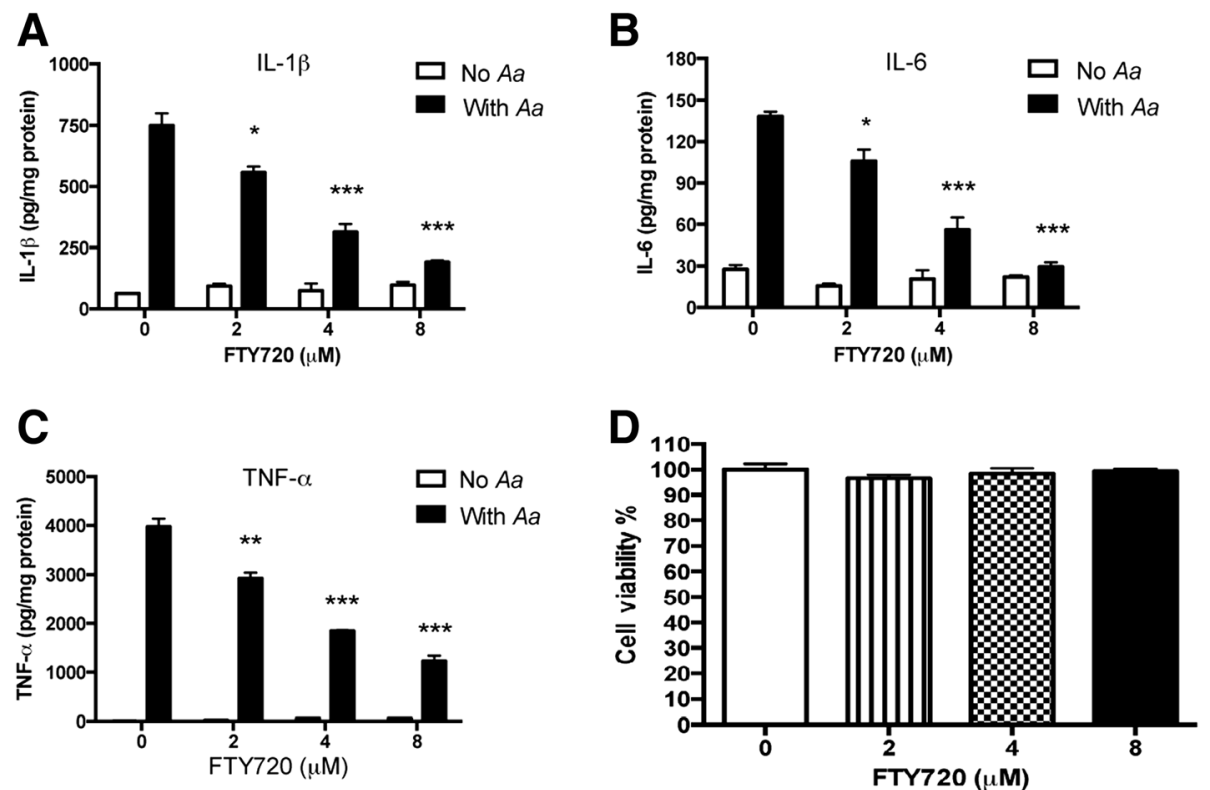

Fig. 1 FTY720 dose-dependently inhibited IL-1 $\beta$, IL-6, and TNF-a expressions induced by A. actinomycetemcomitans in BMMs. Murine BMMs were treated with vehicle (ethanol) or FTY720 (2 to $8 \mu \mathrm{M})$ for $30 \mathrm{~min}$. Then the cells were either unstimulated or stimulated for $6 \mathrm{~h}$ with $A$. actinomycetemcomitans ( $A a)$ (1.5 CFU/cell). a IL-1 $\beta$, (b) IL-6, and (c) TNF-a protein levels in the cell culture media of BMMs were analyzed by ELISA. $\mathbf{d}$ Cell viability was tested in BMMs treated with vehicle or FTY720 ( 2 to $8 \mu \mathrm{M})$ for $8 \mathrm{~h}$. Data are expressed as mean \pm SEM $(n=3$, $\left.{ }^{*} p<0.05,{ }^{* *} p<0.01,{ }^{* *} p<0.001\right)$

$(8 \mu \mathrm{M})$ decreased IL-1 $\beta$ by $74.5 \%$, IL- 6 by $78.7 \%$, and TNF- $\alpha$ by $69.1 \%$ induced by $A$. actinomycetemcomitans compared with the control treatment. FTY720 ( $4 \mu \mathrm{M})$ also reduced IL- $1 \beta$ by $58.3 \%$, IL- 6 by $59.5 \%$, and TNF- $\alpha$ by $53.5 \%$ induced by $A$. actinomycetemcomitans compared with the control treatment. FTY720 ( 2 to $8 \mu \mathrm{M}$ ) did not induce cell death in BMMs $8 \mathrm{~h}$ after treatment (Fig. 1d). These data supported that FTY720 suppressed the proinflammatory cytokine response induced by the oral pathogen $A$. actinomycetemcomitans.

\section{FTY720 attenuated p-PI3K, p-Akt, and p-ERK expressions induced by $A$. actinomycetemcomitans in BMMs}

To further elucidate which signaling pathways were affected by FTY720 in regulating the immune response induced by $A$. actinomycetemcomitans, we performed Western blot assays in BMMs treated with vehicle (ethanol) or FTY720 $(8 \mu \mathrm{M})$, with or without A. actinomycetemcomitans stimulation. As shown in Fig. 2a-d, FTY720 treatment decreased p-PI3K by $92.5 \%$, p-Akt by $65.9 \%$, and p-ERK by $54.0 \% 30 \mathrm{~min}$ after bacterial stimulation compared with the control treatment. FTY720 reduced p-PI3K by $75.2 \%$, p-Akt by $76.9 \%$, and p-ERK by $59.1 \% 60$ min after bacterial stimulation compared with the control treatment. Additionally, FTY720 attenuated p-PI3K by $43.6 \%$, p-Akt by $59.2 \%$, and p-ERK by $50.0 \%$ in cells without bacterial stimulation compared with the control treatment. The protein levels of p-NF-
кB p65, p-JNK, p-p38 MAPK, and glyceraldehyde 3phosphate dehydrogenase (GAPDH) were similar between FTY720-treated cells and vehicle-treated cells before or after bacterial stimulation (data not shown). These results supported that FTY720 specifically attenuated the PI3K, Akt, and ERK signaling pathways, which could contribute to the down-regulation of the proinflammatory cytokine response stimulated by A. actinomycetemcomitans.

\section{FTY720 suppressed osteoclastogenesis in bone marrow-derived pre-osteoclasts with or without bacterial stimulation}

Osteoclasts originate from the fusion of monocytes and macrophages [27]. It has been recognized that phosphoinositides signaling controls the activation of Nfatc1 and influences osteoclastogenesis [28]. Furthermore, proinflammatory cytokines promote the differentiation of osteoclasts [1]. Since FTY720 significantly inhibited PI3K signaling and attenuated proinflammatory cytokine expression induced by $A$. actinomycetemcomitans, we hypothesized that FTY720 could further inhibit osteoclastogenesis. To test our hypothesis, murine bone marrow cells were treated with M-CSF $(50 \mu \mathrm{g} / \mathrm{mL})$ for two days to allow bone marrow progenitor cells to differentiate into bone marrow-derived pre-osteoclasts. To determine if FTY720 could inhibit osteoclastogenesis induced by RANKL, bone marrow-derived pre-osteoclasts were treated with M-CSF $(50 \mu \mathrm{g} / \mathrm{mL})$ and RANKL $(100 \mathrm{ng} / \mathrm{mL})$ for three days; then 

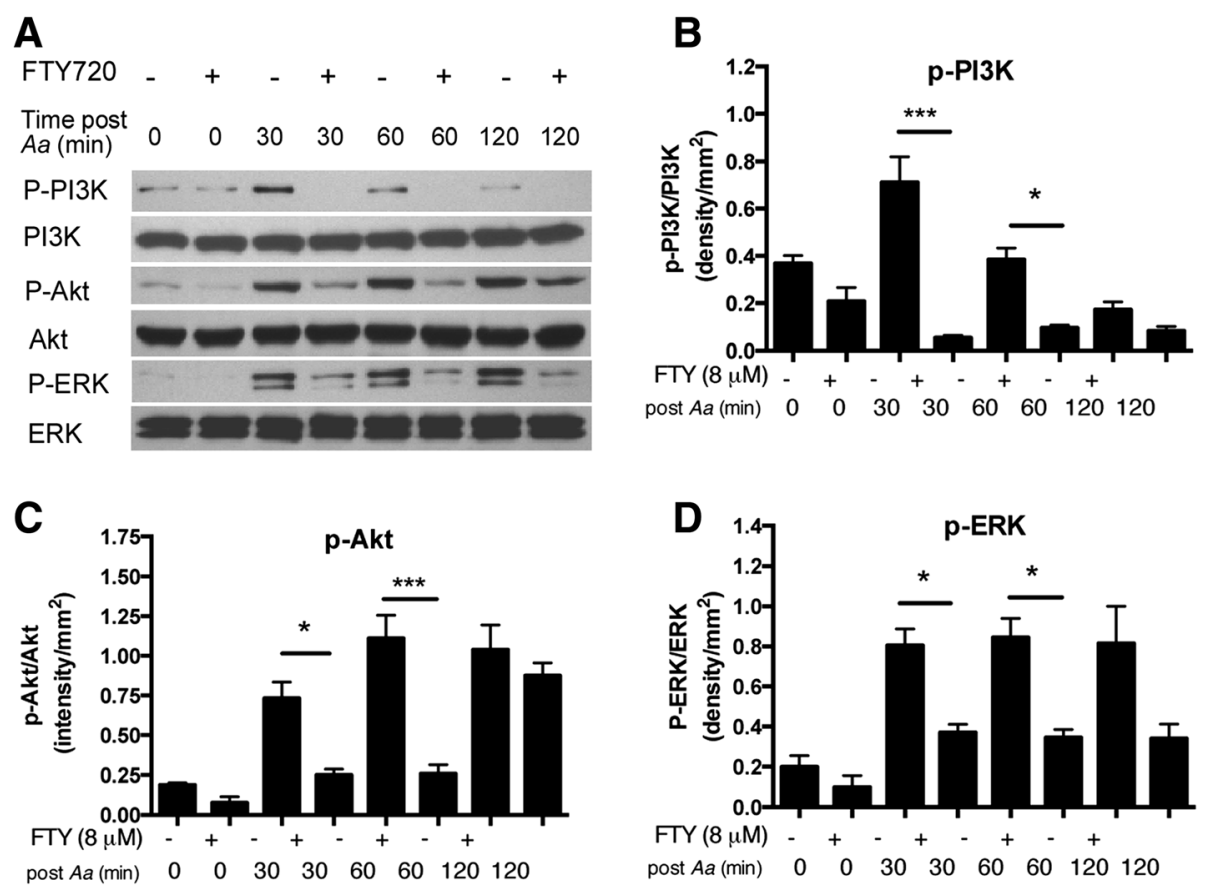

Fig. 2 FTY720 attenuated p-PI3K, p-Akt, and p-ERK expressions induced by A. actinomycetemcomitans in BMMs. Murine BMMs were treated with vehicle (ethanol) or FTY720 (8 $\mu \mathrm{M})$ for $30 \mathrm{~min}$. Then the cells were either unstimulated or stimulated with A. actinomycetemcomitans (Aa) (1.5 CFU/cell) for 30 to 120 min. a P-PI3K, PI3K, p-Akt, Akt, p-ERK, and ERK expressions were evaluated by Western blot. b P-PI3K protein density, (c) P-Akt protein density, and (d) P-ERK protein density were analyzed by Quantity One Software and normalized by total protein expression, respectively. Data are expressed as mean \pm $\operatorname{SEM}\left(n=3,{ }^{*} p<0.05,{ }^{* * *} p<0.001\right)$

the media were changed with fresh media containing $\mathrm{M}$ CSF $(50 \mu \mathrm{g} / \mathrm{mL})$ and RANKL $(100 \mathrm{ng} / \mathrm{mL})$. Cells were treated with FTY720 $(2 \mu \mathrm{M})$ or vehicle (ethanol) for $24 \mathrm{~h}$ (Fig. 3a). Additionally, to determine if FTY720 could attenuate osteoclastogenesis induced by $A$. actinomycetemcomitans, bone marrow-derived pre-osteoclasts were treated with M-CSF $(50 \mu \mathrm{g} / \mathrm{mL})$ and RANKL $(100 \mathrm{ng} / \mathrm{mL})$ for three days. To reduce the background of osteoclastogenesis induced by RANKL, the media were changed with media containing only M-CSF $(50 \mu \mathrm{g} / \mathrm{mL})$ without RANKL. The cells were treated for $30 \mathrm{~min}$ with vehicle or FTY720 $(2 \mu \mathrm{M})$. Then the cells were either unstimulated or stimulated for $24 \mathrm{~h}$ with $A$. actinomycetemcomitans (0.5 CFU/cell), in the presence of FTY720 or vehicle (Fig. 3a). Control cells were treated with media containing only M-CSF $(50 \mu \mathrm{g} / \mathrm{mL})$ with or without bacterial stimulation. Osteoclasts were detected by tartrate-resistant acid phosphatase (TRAP) staining $24 \mathrm{~h}$ after FTY720 or vehicle treatment. As shown in Fig. 3b, there were no $\mathrm{TRAP}^{+}$osteoclasts in cells treated with only M-CSF with or without bacterial stimulation. There were many $\mathrm{TRAP}^{+}$multinucleated osteoclasts in cells treated with vehicle in the presence of both M-CSF and RANKL with or without bacterial stimulation. In contrast, FTY720 $(2 \mu \mathrm{M})$ treatment decreased both size and number of $\mathrm{TRAP}^{+}$multinucleated osteoclasts compared with vehicle groups. Quantification of the number of osteoclasts showed that there was a 1.9-fold increase of the number of osteoclasts in cells treated with M-CSF and RANKL stimulated with A. actinomycetemcomitans compared with cells treated with M-CSF and RANKL without bacterial stimulation (Fig. 3c). FTY720 treatment reduced the number of osteoclasts by $53.2 \%$ in cells treated with M-CSF and RANKL without bacterial stimulation, and decreased the number of osteoclasts by $64.3 \%$ in cells treated with M-CSF and RANKL with bacterial stimulation, as compared with vehicle controls, respectively (Fig. 3c). Quantification of total area of osteoclasts per image revealed that FTY720 reduced the area of osteoclasts by $74.2 \%$ in cells treated with M-CSF and RANKL without bacterial stimulation, and FTY720 decreased the area of osteoclasts by $71.4 \%$ in cells treated with M-CSF and RANKL with bacterial stimulation (Fig. 3d). FTY720 $(2 \mu \mathrm{M})$ treatment for $24 \mathrm{~h}$ did not induce cell death in bone marrow-derived pre-osteoclast (Fig. 3e). These data demonstrated that FTY720 suppressed osteoclastogenesis in bone marrow-derived pre-osteoclasts treated with M-CSF and RANKL with or without bacterial stimulation. 


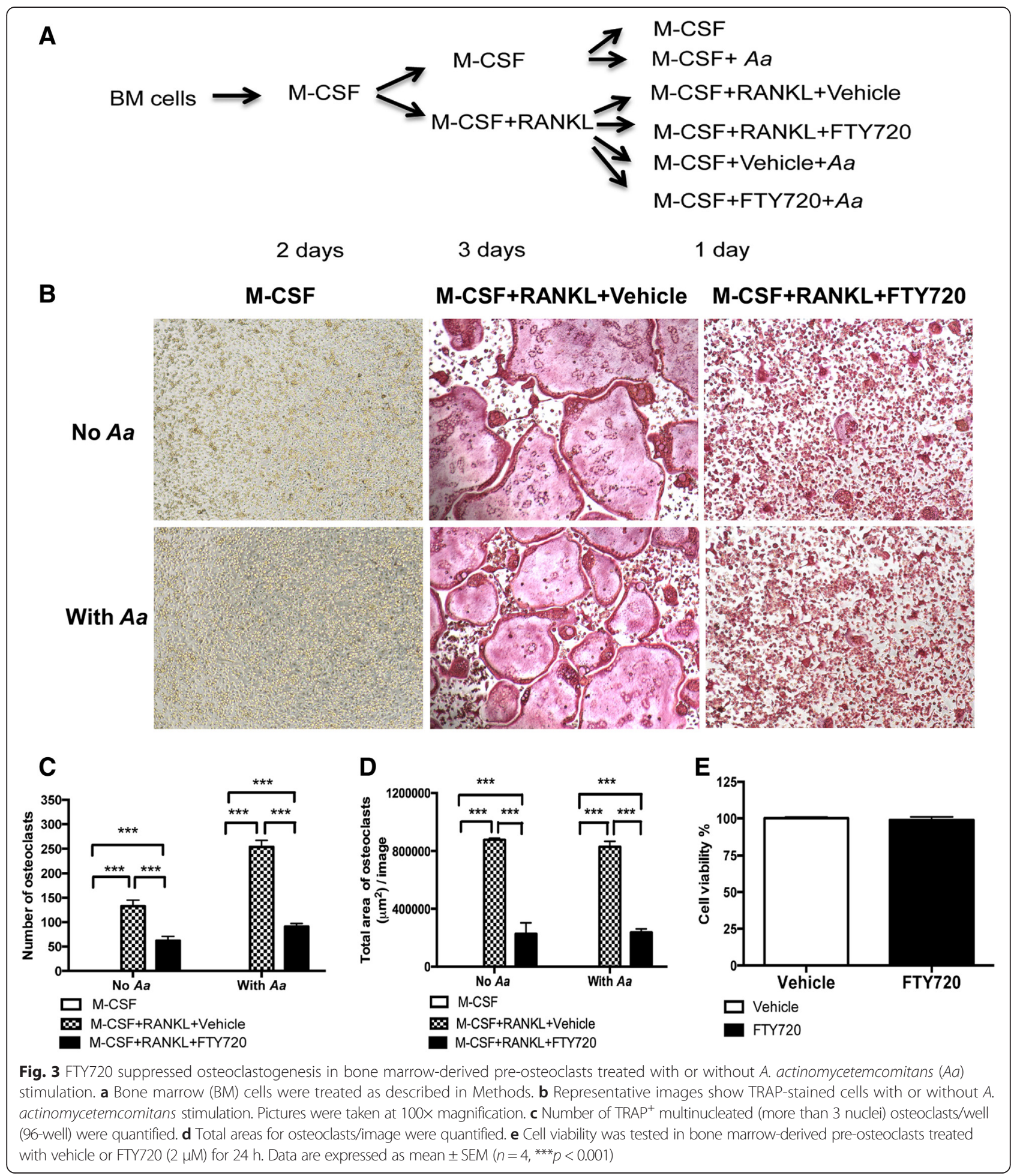

FTY720 significantly decreased the mRNA expressions of Nfatc1, Ctsk, Acp5, and Oscar in bone marrow-derived pre-osteoclasts with or without bacterial stimulation To elucidate the mechanisms associated with the role of FTY720 in osteoclastogenesis, we analyzed the mRNA expressions of various kinds of osteoclastogenic factors, including Nfatc1, Ctsk, Acp5, Oscar, and RANKL in bone marrow-derived pre-osteoclasts treated for 4 or for 24 h with FTY720 $(2 \mu \mathrm{M})$ or vehicle (ethanol), with or without $A$. actinomycetemcomitans stimulation. As shown in Fig. 4a-d, cells treated with both M-CSF and RANKL significantly increased the mRNA levels of Nfatc1, Ctsk, Acp5, 

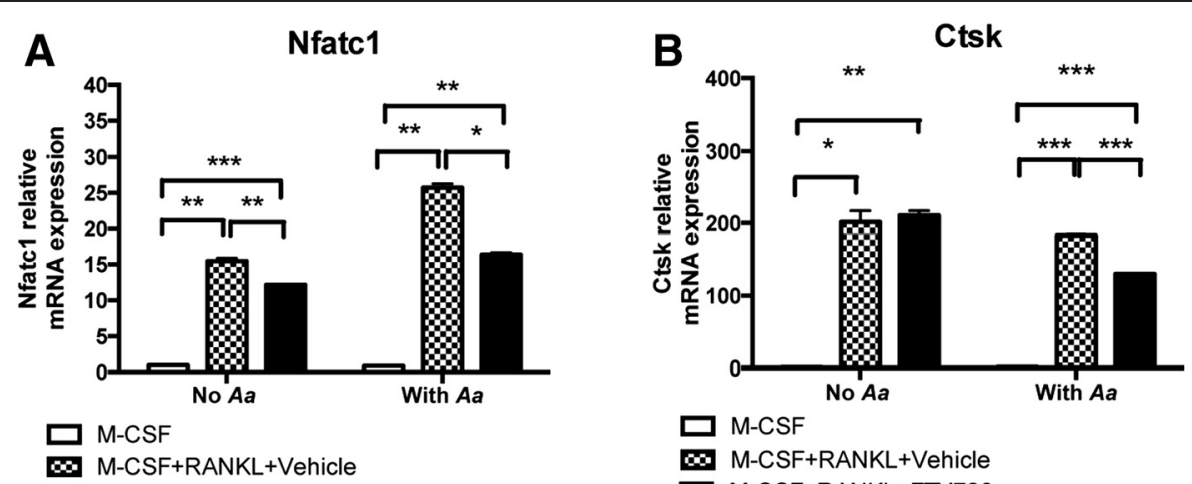

$\square \mathrm{M}-\mathrm{CSF}$

D. M-CSF+RANKL+Vehicle

M-CSF+RANKL+FTY720

M-CSF+RANKL+FTY720

C

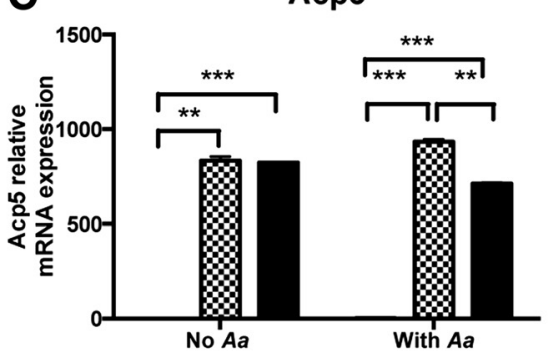

$\square \mathrm{M}-\mathrm{CSF}$

M-CSF+RANKL+Vehicle

M-CSF+RANKL+FTY720

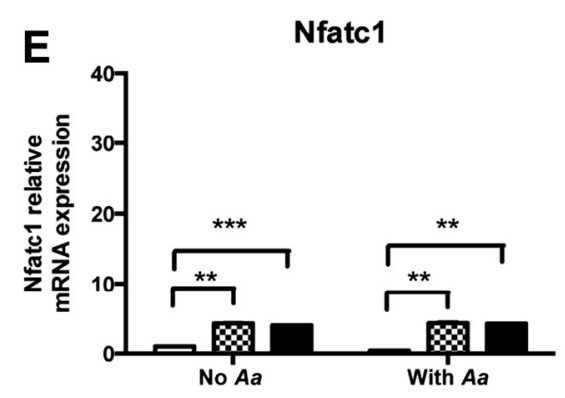

$\square \mathrm{M}-\mathrm{CSF}$

M-CSF+RANKL+Vehicle

M-CSF+RANKL+FTY720

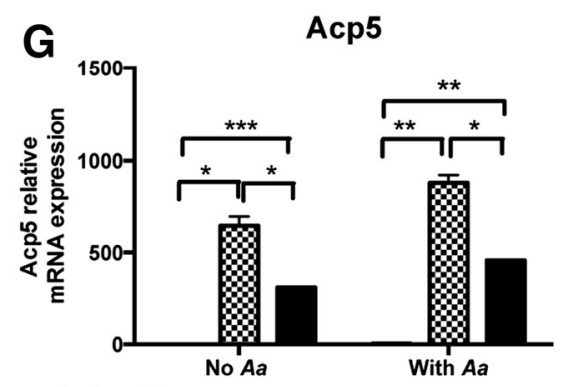

$\square \mathrm{M}-\mathrm{CSF}$

a. M-CSF+RANKL+Vehicle

M-CSF+RANKL+FTY720
D

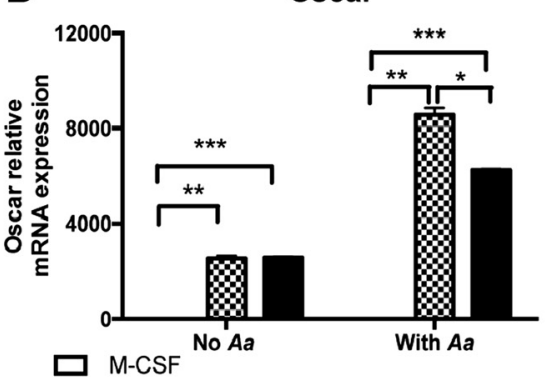

W M-CSF+RANKL+Vehicle

M-CSF+RANKL+FTY720

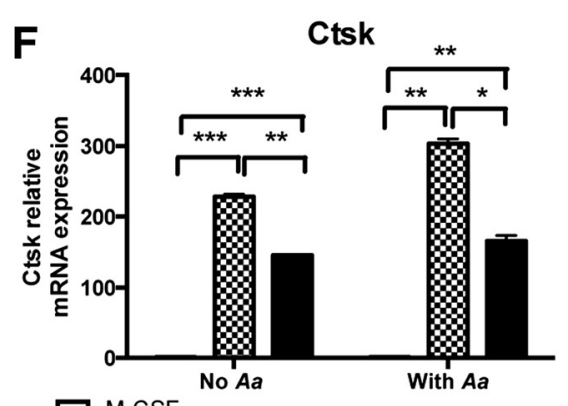

$\square$ M-CSF

M-CSF+RANKL+Vehicle

M-CSF+RANKL+FTY720

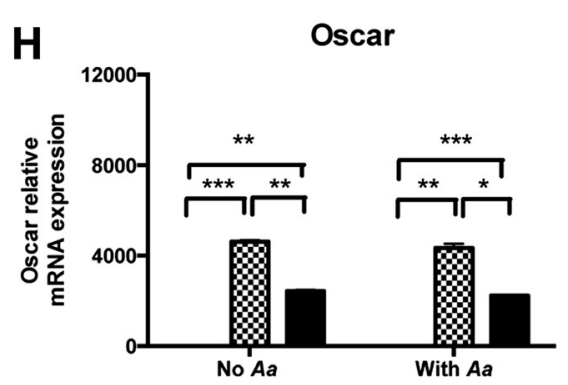

$\square \mathrm{M}-\mathrm{CSF}$

M-CSF+RANKL+Vehicle

M-CSF+RANKL+FTY720

Fig. 4 (See legend on next page.) 
(See figure on previous page.)

Fig. 4 FTY720 significantly decreased Nfatc1, Ctsk, Acp5, and Oscar expressions in bone marrow-derived pre-osteoclasts with or without A. actinomycetemcomitans $(\mathrm{Aa}$ ) stimulation. Bone marrow-derived pre-osteoclasts were treated as described in Methods. Cells were treated with vehicle (ethanol) or FTY720 (2 $\mu \mathrm{M})$ for $30 \mathrm{~min}$. Then the cells were either unstimulated or stimulated with A. actinomycetemcomitans (Aa) $(0.5$ CFU/cell) in the presence of vehicle or FTY720 for $4 \mathrm{~h}$ (a-d) or for $24 \mathrm{~h}$ (e-h). a, e Nfatc1 mRNA, (b, f) Ctsk mRNA, (c, g) Acp5 mRNA, and $(\mathbf{d}, \mathbf{h})$ Oscar mRNA levels were normalized by an endogenous control GAPDH expression and expressed as fold change compared with control group. Data are expressed as mean $\pm \operatorname{SEM}\left(n=3,{ }^{*} p<0.05,{ }^{* *} p<0.01,{ }^{* * *} p<0.001\right)$

and Oscar compared with those levels in cells treated with M-CSF alone. In cells treated with FTY720 for $4 \mathrm{~h}$ without bacterial stimulation (Fig. 4a), there was a $22.0 \%$ significant reduction of Nfatc1 mRNA expression as compared with vehicle control. However, there were no significant differences of the mRNA levels of Ctsk, Acp5, and Oscar between FTY720-treated cells and vehicle-treated cells (Fig. 4b-d). In cells treated with FTY720 for $24 \mathrm{~h}$ without bacterial stimulation (Fig. 4. e-h), FTY720 significantly decreased the mRNA levels of Ctsk by $36.1 \%$, Acp 5 by $51.5 \%$, and Oscar by $47.3 \%$ compared with those levels in vehicle-treated cells. However, there was no significant difference in Nfatc1 mRNA expression between these two groups.

In cells treated with vehicle and stimulated with $A$. actinomycetemcomitans for $4 \mathrm{~h}$ (Fig. 4a-d), there was a 1.8-fold increase of Nfatc1 and a 3.6-fold increase of Oscar mRNA expression compared with those in cells without bacterial stimulation. FTY720 significantly reduced the mRNA levels of Nfatc1 by $36.5 \%$, Ctsk by $28.9 \%$, Acp 5 by $23.6 \%$, and Oscar by $27.0 \%$ compared with vehicle controls. In cells treated with vehicle stimulated with bacteria for $24 \mathrm{~h}$ (Fig. $4 \mathrm{e}-\mathrm{h}$ ), we observed a 1.3-fold increase of Ctsk and a 1.6-fold increase of Acp5 mRNA levels compared with those levels in cells treated with M-CSF and RANKL without bacterial stimulation. FTY720 significantly decreased the mRNA levels of Ctsk by $45.4 \%$, Acp 5 by $47.7 \%$, and Oscar by $48.5 \%$ compared with vehicle controls. However, there was no significant difference in Nfatc1 mRNA expression at $24 \mathrm{~h}$ between FTY720 treated-cells and vehicle-treated cells. These data supported that FTY720 decreased the mRNA expressions of osteoclastogenic factors, including Nfatc1, Ctsk, Acp5, and Oscar in bone marrow-derived preosteoclasts treated with M-CSF and RANKL with or without bacterial stimulation, which subsequently influenced the osteoclastogenesis.

\section{Discussion}

Previous studies showed that FTY720, a modulator of multiple S1P receptors, exerted immunosuppressive activity and attenuated bone loss in mice [7, 18-20]. However, the mechanisms associated with the roles of FTY720 on modulating inflammatory diseases and bone loss diseases have not yet been completely elucidated. In this in vitro study, we demonstrated that FTY720 suppressed PI3K-Akt and ERK signaling pathways and attenuated IL-1 $\beta$, IL- 6 , and TNF- $\alpha$ expressions induced by $A$ actinomycetemcomitans. Importantly, we first demonstrated that FTY720 suppressed osteoclastogenesis in bone marrow-derived pre-osteoclasts. Mechanistically, we demonstrated that FTY720 inhibited the expressions of osteoclastogenic factors, including Nfatc1, Ctsk, Acp5, and Oscar. Our study suggested that S1PR signaling pathways might be involved in the modulation of proinflammatory response induced by bacterial stimulation and may affect the osteoclastogenesis.

Previously, FTY720 was shown to exhibit an immunosuppressive effect in vivo [18, 19]. Early studies demonstrated that this effect was mainly caused by internalization and down-regulation of S1PR1 by FTY720 [26, 29], which resulted in the suppression of the egress of mature lymphocytes from the secondary lymphoid organs to peripheral blood and lymph [30]. In the current in vitro study, we demonstrated that FTY720 had a direct anti-inflammatory effect by suppressing IL- $1 \beta$, IL- 6 and TNF- $\alpha$ expression induced by the oral pathogen $A$. actinomycetemcomitans. This might be caused by the down-regulation of PI3K, Akt, and ERK signaling pathways by FTY720 in BMMs. Previous studies showed that human peripheral blood mononuclear cells (PBMCs) treated with a PI3K inhibitor, wortmannin, significantly inhibited LPS-induced chemokine C-X-C motif ligand 8 (CXCL8) and IL-6 release [31]. Additionally, PBMCs treated with pertussis toxin, a small $G$ protein inhibitor, inhibited LPS-induced Akt phosphorylation and reduced the generation of CXCL8 and IL-6 [31]. Furthermore, murine macrophages treated with trametinib, a highly potent ERK inhibitor, significantly reduced LPSinduced TNF- $\alpha$ mRNA and protein secretion [32]. Since PI3K, Akt, and ERK signaling pathways were up-regulated by bacterial stimulation, and activation of these signaling pathways contributed to the production of proinflammatory cytokines, suppression of these signaling pathways by FTY720 might subsequently reduce IL-1 $\beta$, IL- 6 and TNF- $\alpha$ expressions induced by bacterial stimulation. Previously, Noda et al. [33] also demonstrated that FTY720 inhibited the production of LPS-induced proinflammatory cytokine IL- $1 \beta$, IL-6, and TNF- $\alpha$ in microglia. In accordance with Noda et al., our study demonstrated that FTY720 suppressed IL-1 $\beta$, IL-6 and TNF- $\alpha$ expressions induced by $A$. actinomycetemcomitans. 
Although it is known that FTY720 is a modulator for multiple S1PRs, researchers continue to debate as to which S1PRs are regulated by p-FTY720. Early studies supported that p-FTY720 bound with higher affinity with S1PR1, 3, 4 and 5, and served as a S1P agonist [34, 35]. However, later studies demonstrated that p-FTY720 functioned as a noncompetitive inhibitor of multiple S1PRs [16, 17]. Graler et al. [16] demonstrated that FTY720 blocked S1P signaling through S1PR1, 2, and 5 by inducing their internalization and intracellular partial degradation without affecting S1PR3 or S1PR4. Another study showed that human monocyte-derived dendritic cells treated with both FTY720 and p-FTY720 resulted in decreased S1PR1 and S1PR4 levels [17]. Future studies need to determine which of the five S1PRs might play a role in regulating the inflammatory response stimulated by $A$. actinomycetemcomitans.

In addition to modulating inflammatory response, previous studies demonstrated that S1P signaling was critical in modulating bone homeostasis [7, 14]. Lee et al. [14] showed that S1P levels were significantly higher in postmenopausal women than those in the premenopausal women and men, and the higher S1P levels in postmenopausal women were positively correlated with their bone resorption marker. In contrast, blocking S1P signaling by FTY720 inhibited osteoporosis in mice with ovariectomy [7]. Ishii et al. [7] explained that the anti-osteoporotic role of FTY720 is mainly caused by the inhibition of the migration of osteoclast precursors from the circulation to bone tissues. In the current in vitro assay, we demonstrated that FTY720 suppressed the differentiation of osteoclasts and attenuated the expressions of osteoclastogenic factors, including Nfatc1, Ctsk, Acp5, and Oscar.

Osteoclastogenesis involves fusion of osteoclast precursors to form multinucleated mature osteoclasts. It has been recognized that membrane lipids, especially phosphoinositides, are key signaling molecules that regulate osteoclastogenesis [28]. Activation of PI3K triggers the $\mathrm{Ca}^{2+}$ release followed by activation of Nfatc1, a master transcription factor for osteoclastogenic gene regulation $[23,28]$. In this study, we demonstrated that FTY720 attenuated p-PI3K levels in BMMs before or after bacterial stimulation compared with vehicle treatment. Previously, Graler et al. [16] showed that $\mathrm{HTC}_{4}$ cells (rat hepatoma cells) treated with FTY720 decreased calcium release. We observed significantly decreased mRNA levels of Nfatc1, Ctsk, Acp5, and Oscar in FTY720-treated cells with or without bacterial stimulation compared with those in the vehicle-treated cells. The down-regulation of these osteoclastogenic factors by FTY720 might be associated with the decreased p-PI3K and possibly reduced intracellular calcium levels in FTY720-treated cells before or after bacterial stimulation. Since RANKL induces osteoclastogenesis via activation of Nfatc1 [36], down-regulation of Nfatc1 by FTY720 could inhibit osteoclastogenesis induced by RANKL without bacterial stimulation. In addition, reducing IL-1 $\beta$, IL- 6 and TNF- $\alpha$ expressions induced by bacterial stimulation by FTY720 could further attenuate osteoclastogenesis. In this study, we observed significant reductions of Nfatc1 mRNA levels by FTY720 at $4 \mathrm{~h}$ after treatment compared with vehicle controls, while we did not observe this significant reduction of Nfatc1 mRNA levels at $24 \mathrm{~h}$ in FTY720-treated cells compared with vehicle controls with or without bacterial stimulation. This suggests that activation of Nfatc1 is an early event that might occur before the activation of Ctsk, Acp 5 and Oscar.

A previous study showed that low doses of FTY720 (20 to $100 \mathrm{nM}$ ) did not inhibit osteoclastogenesis in BMMs treated with RANKL and M-CSF for 4 days [13]. In this study, FTY720 $(2 \mu \mathrm{M})$ suppressed osteoclastogenesis in bone marrow-derived pre-osteoclasts treated with M-CSF and RANKL with or without bacterial stimulation. Our study suggested that it might require higher doses of FTY720 $(\geq 2 \mu \mathrm{M})$ to suppress p-PI3K and Nfatc1 expressions. Ryu et al. [13] demonstrated that S1P enhanced the expression of RANKL in osteoblasts, and FTY720 (10 nM) was potent to suppress S1P-induced RANKL expression in osteoblasts. In addition, they showed that FTY720 (10 nM) inhibited osteoclastogenesis induced by S1P in a co-culture of BMMs and osteoblasts [13]. Because RANKL is mainly produced in osteoblasts and mesenchymal stem cells, we did not observe a significant difference in RANKL expression between FTY720-treated cells and vehicle-treated cells in this single culture of bone marrow-derived preosteoclasts. As FTY720 is a modulator of multiple S1PRs, future studies need to determine which of the five S1PRs play a major role in regulating PI3K pathway, calcium release, and the expressions of various osteoclastogenic factors, including RANKL, Nfatc1, Ctsk, Acp5, and Oscar.

\section{Conclusions}

FTY720 inhibited proinflammatory cytokine production in BMMs and suppressed osteoclastogenesis in bone marrow-derived pre-osteoclasts with or without A. actinomycetemcomitans stimulation, supporting FTY720 as a potential therapy for inflammatory bone loss diseases.

\section{Methods}

Murine bone marrow-derived monocytes and macrophages (BMMs) and reagents

Six to eight-week-old male C57BL/6 mice were purchased from Jackson Laboratory (Bar Harbor, ME, USA). Bone marrow cells were harvested from the femurs and tibias of mice. Murine bone marrow cells were cultured 
for $18 \mathrm{~h}$ in tissue culture dishes in complete MEM- $\alpha$ media (Life Technologies, Grand Island, NY, USA) containing $10 \%$ fetal bovine serum (FBS), $100 \mathrm{U} / \mathrm{mL}$ penicillin, and $100 \mu \mathrm{g} / \mathrm{mL}$ streptomycin to remove adherent cells. To allow bone marrow progenitor cells to differentiate into BMMs, non-adherent cells were transferred to new tissue culture dishes and cultured for 7 days in complete MEM- $\alpha$ media supplemented with $50 \mathrm{ng} / \mathrm{mL}$ murine recombinant M-CSF (R\&D systems, Minneapolis, MN, USA). After seven days, the suspended cells were discarded and the attached BMMs were plated in tissue culture dishes. FTY720 was obtained from Santa Cruz biotechnology (Dallas, TX, USA), diluted in ethanol $(10 \mathrm{mM})$, and stored at $-20^{\circ} \mathrm{C}$.

\section{Culture of $A$. actinomycetemcomitans}

A. actinomycetemcomitans (ATCC 43718, serotype b, strain Y4) was purchased from American Type Culture Collection (Manassas, VA, USA). Bacterial colonies were grown on Difco $^{\text {tm }}$ brain heart infusion agar plates (BD Biosciences, Sparks, MD, USA) and cultured in Bacto ${ }^{\text {Tu }}$ brain heart infusion broth (BD Biosciences) at $37{ }^{\circ} \mathrm{C}$ with $10 \% \mathrm{CO}_{2}$ for $24 \mathrm{~h}$. Bacteria were centrifuged, washed with PBS with $5 \%$ glycerol, and resuspended in PBS with $5 \%$ glycerol. Bacterial concentration was determined by measuring bacterial optical density and followed by plating on brain heart infusion agar plates $\left(\mathrm{OD}_{600}=1\right.$, about $3 \times 10^{7}$ colony forming unit, $\mathrm{CFU} / \mathrm{mL})$.

\section{Enzyme-linked immunosorbent assay (ELISA)}

IL-1 $\beta$, IL- 6 , and TNF- $\alpha$ cytokine levels in the cell culture media of BMMs were quantified by ELISA kits (R\& D Systems). The protein concentrations from cell lysates were determined by a DC protein Assay Kit (Bio-Rad Laboratories, Hercules, CA, USA). The concentration of cytokines was normalized by protein concentration in cell lysates.

\section{Cell viability assay}

Bone marrow cells $\left(1 \times 10^{5} /\right.$ well $)$ in a 96-well plate were incubated with either vehicle (ethanol) or FTY720 (2 to $8 \mu \mathrm{M})$ for 8 to $24 \mathrm{~h}$. The cell viability was analyzed by CellTiter 96 Aqueous One Solution Cell Proliferation Assay (Promega, Madison, WI, USA).

\section{Western blot assay}

BMMs were lysed in RIPA protein lysis buffer (Cell Signaling Technology, Danvers, MA, USA). Western blot was performed as previously described [37]. The antibodies to p-PI3K, PI3K, p-Akt, Akt, p-ERK, ERK, p-JNK, p-p38, p-NF-кBp65, and GAPDH were purchased from Cell Signaling Technology (Danvers, MA, USA).

\section{Osteoclastogenesis assay and tartrate-resistant acid phosphatase (TRAP) staining}

Murine bone marrow cells were cultured for $18 \mathrm{~h}$ in tissue culture dishes in complete MEM- $\alpha$ media to remove adherent cells. Non-adherent cells were transferred to new tissue culture dishes and cultured for 2 days in complete MEM- $\alpha$ media, supplemented with $50 \mathrm{ng} / \mathrm{mL}$ murine recombinant M-CSF to allow cells to differentiate into pre-osteoclasts. At day 3, cells were plated at a density of $1 \times 10^{6}$ cells/well in 12 -well plates or $1.5 \times 10^{5}$ cells/well in 96-well plate, and cultured in complete MEM- $\alpha$ media, supplemented with either $50 \mathrm{ng} / \mathrm{mL}$ murine recombinant M-CSF alone (control) or with both $50 \mathrm{ng} / \mathrm{mL} \mathrm{M}-\mathrm{CSF}$ and $100 \mathrm{ng} / \mathrm{mL}$ RANKL (R\&D Systems). At day 6, the media were changed and the cells were cultured in fresh MEM- $\alpha$ media containing $50 \mathrm{ng} / \mathrm{mL}$ M-CSF with or without $100 \mathrm{ng} / \mathrm{mL}$ RANKL. The cells were treated with either FTY720 $(2 \mu \mathrm{M})$ or vehicle (ethanol) for $30 \mathrm{~min}$. Then the cells were either unstimulated or stimulated with A. actinomycetemcomitans $(0.5 \mathrm{CFU} / \mathrm{cell})$ in the presence of FTY720 or vehicle for $4 \mathrm{~h}$ or for $24 \mathrm{~h}$. Control cells were treated with M-CSF alone with or without bacterial stimulation. TRAP staining was performed in the cells using a leukocyte acid phosphatase kit (Sigma Aldrich, St. Louis, MO, USA) $24 \mathrm{~h}$ after FTY720 treatment. Pictures were taken by a Nikon Eclipse TS-100 inverted microscope. Image analysis was performed using Visiopharm 5.0 software (Visiopharm, Hoersholm, Denmark).

\section{RNA extraction and real time polymerase chain reaction (PCR)}

The RNA extraction, reverse transcription, and real time PCR were performed as previously described [2]. The following amplicon primers were obtained from Life Technologies: Nfatc1 (Mm00479445_m1), Ctsk (Mm00484039_m1), Acp5 (Mm00475698_m1), Oscar (Mm00558665_m1), RANKL (Mm00441906_m1), and GAPDH (Mm99999915_g1). Amplicon concentration was determined using threshold cycle values compared with standard curves for each primer. Sample mRNA levels were normalized to an endogenous control GAPDH expression and expressed as fold changes compared with control groups.

\section{Statistical analyses}

All experiments were performed in triplicate with bone marrow cells from mice. Data were analyzed by one-way ANOVA with Holm-Sidak's multiple comparisons test. All statistical tests were performed using GraphPad Prism software (GraphPad Software Inc., La Jolla CA, USA). Values are expressed as mean \pm standard error of the mean (SEM). A $P$ value of 0.05 or less was considered significant. 


\section{Abbreviations}

A. actinomycetemcomitans: Aggregatibacter actinomycetemcomitans; Acp5: Acid phosphatase 5; BMM: Bone marrow-derived monocytes and macrophages; CFU: Colony forming unit; Ctsk: Cathepsin K; ELISA: Enzymelinked immunosorbent assay; ERK: Extracellular signal-regulated kinase; FBS: Fetal bovine serum; GAPDH: Glyceraldehyde 3-phosphate dehydrogenase; IL: Interleukin; JNK: c-Jun N-terminal kinase; MAPK: Mitogen-activated protein kinase; Nfatc1: Nuclear factor of activated T-cells cytoplasmic calcineurindependent 1; M-CSF: Macrophage colony-stimulating factor, NF-KB, nuclear factor-kappaB; Oscar: Osteoclast-associated receptor; PCR: Polymerase chain reaction; PI3K: Phosphoinositide 3-kinase RANKL: Receptor activator of nuclear factor kappa-B ligand; SEM: Standard error of the mean; S1P: Sphingosine-1-phosphate; S1PR: Sphingosine-1-phosphate receptor; TRAP: Tartrate-resistant acid phosphatase.

\section{Competing interests}

The authors declare that they have no competing interests.

\section{Authors' contributions}

The author HY designed, performed the experiments, and wrote the first draft of the manuscript. $\mathrm{BH}$ and $\mathrm{MV}$ analyzed the data and revised the manuscript. LY and L-C H contributed to data collection. KA reviewed and revised the manuscript. All authors have approved the final manuscript.

\section{Acknowledgment}

This study was supported by grant P30 GM103339, R03 DE025026 from the National Institutes of Health. In addition, this project utilized the facility and resources of the Laboratory of the Center for Oral Health Research (L-COHR), which is supported by the National Institute of General Medicine grant P30 GM103331. We thank the Writing Center at Medical University of South Carolina for manuscript review and assistance. The authors declare no potential conflicts of interest with respect to the authorship and/or publication of this article.

\section{Author details}

'Department of Oral Health Sciences and the Center for Oral Health Research, Medical University of South Carolina, 173 Ashley Avenue, Charleston, SC 29425, USA. ${ }^{2}$ Clemson University, Clemson, SC 29634, USA. ${ }^{3}$ Department of Regenerative Medicine and Cell Biology, Medical University of South Carolina, 173 Ashley Avenue, Charleston, SC 29425, USA

\section{Received: 2 February 2015 Accepted: 12 June 2015}

Published online: 04 July 2015

\section{References}

1. Graves D. Cytokines that promote periodontal tissue destruction. J Periodontol. 2008;79:1585-91.

2. $\mathrm{Yu} \mathrm{H}$, Valerio M, Bielawski J. Fenretinide inhibited de novo ceramide synthesis and proinflammatory cytokines induced by Aggregatibacter actinomycetemcomitans. J Lipid Res. 2013;54:189-201.

3. Xia P, Wadham C. Sphingosine 1-phosphate, a key mediator of the cytokine network: juxtacrine signaling. Cytokine Growth Factor Rev. 2011;22:45-53.

4. Spiegel S, Milstien S. The outs and the ins of sphingosine-1-phosphate in immunity. Nat Rev Immunol. 2011;11:403-15.

5. Snider AJ, Orr Gandy KA, Obeid LM. Sphingosine kinase: Role in regulation of bioactive sphingolipid mediators in inflammation. Biochimie. 2010;92:707-15.

6. Takabe K, Paugh SW, Milstien S, Spiegel S. "Inside-out" signaling of sphingosine-1phosphate: therapeutic targets. Pharmacol Rev. 2008:60:181-95.

7. Ishii M, Egen JG, Klauschen F, Meier-Schellersheim M, Saeki Y, Vacher J, et al. Sphingosine-1-phosphate mobilizes osteoclast precursors and regulates bone homeostasis. Nature. 2009:458:524-8.

8. Ishii M, Kikuta J. Sphingosine-1-phosphate signaling controlling osteoclasts and bone homeostasis. Biochim Biophys Acta. 1831;2013:223-7.

9. Maceyka M, Harikumar KB, Milstien S, Spiegel S. Sphingosine-1-phosphate signaling and its role in disease. Trends Cell Biol. 2012;22:50-60.

10. Kunkel GT, Maceyka M, Milstien S, Spiegel S. Targeting the sphingosine-1phosphate axis in cancer, inflammation and beyond. Nat Rev Drug Discov. 2013;12:688-702
11. Aarthi JJ, Darendeliler MA, Pushparaj PN. Dissecting the role of the S1P/ S1PR axis in health and disease. J Dent Res. 2011:90:841-54.

12. Lai WQ, Chia FL, Leung BP. Sphingosine kinase and sphingosine-1-phosphate receptors: novel therapeutic targets of rheumatoid arthritis? Future Med Chem. 2012:4:727-33.

13. Ryu J, Kim HJ, Chang EJ, Huang H, Banno Y, Kim HH. Sphingosine 1phosphate as a regulator of osteoclast differentiation and osteoclastosteoblast coupling. EMBO J. 2006:25:5840-51.

14. Lee SH, Lee SY, Lee YS, Kim BJ, Lim KH, Cho EH, et al. Higher circulating sphingosine 1-phosphate levels are associated with lower bone mineral density and higher bone resorption marker in humans. J Clin Endocrinol Metab. 2012;97:E1421-8.

15. Fujita $T$, Inoue $K$, Yamamoto $S$, Ikumoto $T$, Sasaki $S$, Toyama $R$, et al Fungal metabolites. Part 11. A potent immunosuppressive activity found in Isaria sinclairii metabolite. J Antibiot (Tokyo). 1994;47:208-15.

16. Graler MH, Goetzl EJ. The immunosuppressant FTY720 down-regulates sphingosine 1-phosphate G-protein-coupled receptors. FASEB J. 2004;18:551-3.

17. Muller H, Hofer S, Kaneider N, Neuwirt H, Mosheimer B, Mayer G, et al. The immunomodulator FTY720 interferes with effector functions of human monocyte-derived dendritic cells. Eur J Immunol. 2005;35:533-45

18. Montalban X, Comi G, O'Connor P, Gold S, de Vera A, Eckert B, et al. Oral fingolimod (FTY720) in relapsing multiple sclerosis: impact on health-related quality of life in a phase II study. Mult Scler. 2011;17:1341-50.

19. Hoitsma AJ, Woodle ES, Abramowicz D, Proot P, Vanrenterghem Y. FTY720 combined with tacrolimus in de novo renal transplantation: 1-year, multicenter, open-label randomized study. Nephrol Dial Transplant. 2011;26:3802-5.

20. Kikuta J, Iwai K, Saeki Y, Ishii M. S1P-targeted therapy for elderly rheumatoid arthritis patients with osteoporosis. Rheumatol Int 2011;31:967-9.

21. Vaananen HK, Laitala-Leinonen T. Osteoclast lineage and function. Arch Biochem Biophys. 2008;473:132-8

22. Feng $\mathrm{W}$, Xia W, Ye Q, Wu W. Osteoclastogenesis and osteoimmunology. Front Biosci (Landmark Ed). 2014;19:758-67.

23. Zhao Q, Wang X, Liu Y, He A, Jia R. NFATc1: functions in osteoclasts. Int J Biochem Cell Biol. 2010;42:576-9.

24. Nemeth K, Schoppet M, Al-Fakhri N, Helas S, Jessberger R, Hofbauer LC, et al. The role of osteoclast-associated receptor in osteoimmunology. J Immunol. 2011:186:13-8.

25. Matsuo K, Irie N. Transcription factors in osteoclast differentiation. Nihon Rinsho. 2005:63:1541-6.

26. Chiba K, Adachi K. Discovery of fingolimod, the sphingosine 1-phosphate receptor modulator and its application for the therapy of multiple sclerosis. Future Med Chem. 2012:4:771-81.

27. Teitelbaum SL. Bone resorption by osteoclasts. Science. 2000;289:1504-8

28. Oikawa T, Kuroda Y, Matsuo K. Regulation of osteoclasts by membrane-derived lipid mediators. Cell Mol Life Sci. 2013;70:3341-53.

29. Matloubian M, Lo CG, Cinamon G, Lesneski MJ, Xu Y, Brinkmann V, et al. Lymphocyte egress from thymus and peripheral lymphoid organs is dependent on S1P receptor 1. Nature. 2004:427:355-60.

30. Chiba K, Yanagawa Y, Masubuchi Y, Kataoka H, Kawaguchi T, Ohtsuki M et al. FTY720, a novel immunosuppressant, induces sequestration of circulating mature lymphocytes by acceleration of lymphocyte homing in rats. I. FTY720 selectively decreases the number of circulating mature lymphocytes by acceleration of lymphocyte homing. J Immunol. 1998;160:5037-44

31. Ngkelo A, Meja K, Yeadon M, Adcock I, Kirkham PA. LPS induced inflammatory responses in human peripheral blood mononuclear cells is mediated through NOX4 and Gialpha dependent PI-3kinase signalling. J Inflamm (Lond). 2012;9:1.

32. Shi-Lin D, Yuan X, Zhan S, Luo-Jia T, Chao-Yang T. Trametinib, a novel MEK kinase inhibitor, suppresses lipopolysaccharide-induced tumor necrosis factor (TNF)-alpha production and endotoxin shock. Biochem Biophys Res Commun. 2015;458:667-73

33. Noda $H$, Takeuchi $H$, Mizuno $T$, Suzumura A. Fingolimod phosphate promotes the neuroprotective effects of microglia. J Neuroimmunol. 2013;256:13-8.

34. Brinkmann V, Davis MD, Heise CE, Albert R, Cottens S, Hof R, et al. The immune modulator FTY720 targets sphingosine 1-phosphate receptors. J Biol Chem. 2002;277:21453-7. Epub 22002 Apr 21419. 
35. Mandala S, Hajdu R, Bergstrom J, Quackenbush E, Xie J, Milligan J, et al. Alteration of lymphocyte trafficking by sphingosine-1-phosphate receptor agonists. Science. 2002;296:346-9.

36. Kim JH, Kim N. Regulation of NFATc1 in Osteoclast Differentiation. J Bone Metab. 2014;21:233-41.

37. Yu H, Li Q, Herbert B, Zinna R, Martin K, Junior CR, et al. Anti-inflammatory effect of MAPK phosphatase-1 local gene transfer in inflammatory bone loss. Gene Ther. 2011;18:344-53.

Submit your next manuscript to BioMed Central and take full advantage of:

- Convenient online submission

- Thorough peer review

- No space constraints or color figure charges

- Immediate publication on acceptance

- Inclusion in PubMed, CAS, Scopus and Google Scholar

- Research which is freely available for redistribution 